\title{
Fauna do solo como bioindicadora da qualidade do solo em cultivos de cana-de- açúcar: um referencial teórico
}

Soil fauna as a bioindicator of soil quality in sugarcane crops: a theoretical framework

La fauna del suelo como bioindicador de la calidad del suelo en cultivos de caña de azúcar: un marco teórico

Recebido: 26/07/2021 | Revisado: 31/07/2021 | Aceito: 04/08/2021 | Publicado: 09/08/2021

Rafaela Martins da Silva
ORCID: https://orcid.org/0000-0002-7733-8208
Universidade Federal de Viçosa, Brasil
E-mail: rfeng.florestal @ gmail.com
Rakiely Martins da Silva
ORCID: https://orcid.org/0000-0001-6914-4721
E-mail: rakiely@ @otmail.com.br
Universidade Estadual do
Jianne Rafaela Mazzini de Souza
ORCID: https://orcid.org/0000-0002-0803-4438
Universidade Federal de Viçosa, Brasil
E-mail: jianne.rafaela@ @hotmail.com
Tatiane Pimentel Gedgeski
ORCID: https://orcid.org/0000-0002-3382-9283
Universidade Federal Rural do Rio de Janeiro, Brasil
E-mail: tatianepgagro@ gmail.com
Sandra Santana de Lima
ORCID: https://orcid.org/0000-0003-3599-8344
Universidade Federal Rural do Rio de Janeiro, Brasil
E-mail: sandra.biologa@ @otmail.com
Eduardo Lima
ORCID: https://orcid.org/0000-0002-9278-5831
E-mail: edusolosrural@ gmail.com

\section{Resumo}

No sistema de colheita mecanizada da cana-de-açúcar, uma quantidade considerável de resíduos vegetais, denominados de palha ou palhada (folhas secas, bainhas, ponteiros e pedaços de colmo), é acumulada no solo. $\mathrm{O}$ acúmulo dessa palhada causa no solo alterações de ordem física e biológica, melhorando a fertilidade do solo sob essas condições. O manejo inadequado dos agroecossistemas conduz à degradação do ambiente edáfico e, por conseguinte, ao detrimento de sua funcionalidade dentro dos sistemas biológicos. Os impactos que ocorrem no solo devido às mudanças de manejo devem ser avaliados, para tal, a fauna edáfica torna-se um excelente bioindicador, devido a sua estreita relação com os processos que advêm do compartimento serapilheira-solo e sua peculiar suscetibilidade à interferência no ambiente, à composição da comunidade da fauna edáfica reflete a funcionalidade do ecossistema e, unido à densidade, pode explanar as alterações decorrentes das interferências antrópicas na cobertura vegetal. As coberturas vegetais e os diferentes sistemas de cultivo, agem diretamente sobre a comunidade da fauna edáfica, reduzindo a densidade e a diversidade das comunidades em relação à um ecossistema natural. Por meio do estudo da fauna edáfica pode-se além de avaliar a qualidade do solo, ter uma maior compreensão do sistema de produção que este solo comporta, visto que a mesma está intimamente relacionada aos processos de ciclagem de nutrientes e decomposição da matéria orgânica na interface solo-planta.

Palavras-chave: Indicador biológico; Fauna do solo; Saccharum spp.

\begin{abstract}
In the mechanized harvesting system of sugar cane, a considerable amount of plant residues, called straw or straw (dry leaves, sheaths, pointers and stems), is accumulated in the soil. The accumulation of this straw causes physical and biological changes in the soil, improving soil fertility under these conditions. The inadequate management of agroecosystems leads to the degradation of the edaphic environment and, consequently, to the detriment of its functionality within biological systems. The impacts that occur on the soil due to changes in management must be evaluated. For this purpose, the edaphic fauna becomes an excellent bioindicator, due to its close relationship with the processes that come from the litter-soil compartment and its peculiar susceptibility to interference in the soil.
\end{abstract}


environment, the composition of the edaphic fauna community reflects the functionality of the ecosystem and, together with the density, can explain the changes resulting from anthropic interference in the vegetation cover. Vegetable coverings and different cultivation systems act directly on the population of the edaphic fauna, reducing the density and diversity of communities in relation to a natural ecosystem. Through the study of edaphic fauna, in addition to assessing soil quality, having a greater understanding of the production system that this soil supports, since it is closely linked to the processes of nutrient cycling and decomposition of organic matter at the interface soil-plant.

Keywords: Biological indicator; Soil fauna; Saccharum spp.

\section{Resumen}

En el sistema de cosecha mecanizada de la caña de azúcar, se acumula en el suelo una cantidad considerable de residuos vegetales, denominados paja o paja (hojas secas, dobladillos, puntas y pedazos de culmo). La acumulación de esta paja provoca cambios físicos y biológicos en el suelo, mejorando la fertilidad del suelo en estas condiciones. El manejo inadecuado de los agroecosistemas conduce a la degradación del medio edáfico y, en consecuencia, en detrimento de su funcionalidad dentro de los sistemas biológicos. Se deben evaluar los impactos que se producen en el suelo por cambios en el manejo, para ello, la fauna edáfica se convierte en un excelente bioindicador, por su estrecha relación con los procesos que surgen del compartimento hojarasca-suelo y su peculiar susceptibilidad a interferencias en medio ambiente, la composición de la comunidad de fauna edáfica refleja la funcionalidad del ecosistema y, junto con la densidad, puede explicar los cambios resultantes de las interferencias antrópicas en la cobertura vegetal. La cobertura vegetal y los diferentes sistemas de cultivo actúan directamente sobre la comunidad de fauna edáfica, reduciendo la densidad y diversidad de comunidades en relación a un ecosistema natural. A través del estudio de la fauna edáfica, es posible, además de evaluar la calidad del suelo, tener un mayor conocimiento del sistema de producción que comprende este suelo, ya que está íntimamente relacionado con los procesos de ciclo de nutrientes y descomposición de materia orgánica en la interfaz suelo-planta.

Palabras clave: Indicador biológico; Fauna del suelo; Saccharum spp.

\section{Introdução}

O desenvolvimento da cultura da cana-de-açúcar no território brasileiro tem sido beneficiado por novas tecnologias, como a colheita mecanizada (sem queima), que conserva uma cobertura de resíduo vegetal (palhada) sobre o solo, tributando para o aumento do potencial produtivo da cana-de-açúcar (Aquino et al., 2018; Schultz et al., 2010). No sistema de colheita mecanizada da cana-de-açúcar, uma quantidade considerável de resíduos vegetais $(15$ t ha-1), denominados de palha ou palhada (folhas secas, bainhas, ponteiros e pedaços de colmo), é acumulada no solo (Carvalho et al., 2017; Cerri et al., 2013), e essa palhada desempenha um papel fundamental na sustentação da biodiversidade do solo, servindo como fonte primária de carbono (energia), protegendo o solo da radiação solar direta, regulando a temperatura e a umidade do solo e fornecendo abrigo e habitat para a fauna do solo (Menandro et al., 2019).

A palha de cana-de açúcar que por muito tempo era eliminada pela queima antes da colheita (prática ainda comum em algumas regiões do país), atualmente com a colheita mecanizada e consequentemente um maior acumulo de palhada, se tornou uma matéria-prima muito valiosa para a produção de etanol de segunda geração e bioeletricidade (Aquino et al., 2018). No entanto, há pouca informação disponível sobre a quantidade de palha necessária para ser deixada no campo para que a produtividade agrícola não seja afetada e uma alta sustentabilidade seja fornecida para o sistema de produção de bioenergia.

O manejo inadequado dos agroecossistemas conduz à degradação do ambiente edáfico e, por conseguinte, em detrimento de sua funcionalidade dentro dos sistemas biológicos (Menandro et al., 2019). Os impactos que ocorrem no solo devido às mudanças de manejo devem ser avaliados, para tal, a fauna edáfica torna-se um excelente bioindicador, uma vez que estes organismos são sensíveis às modificações no ambiente (Franco et al., 2016; Velásquez et al., 2012).

A fauna edáfica comporta organismos invertebrados que vivem apenas ou algumas fases de seu desenvolvimento no solo ou serapilheira (Aquino; Correia, 2005). Esses organismos desempenham uma série de funções como fragmentação de resíduos orgânicos, aeração, ciclagem de nutrientes, decomposição de matéria orgânica e manutenção do equilíbrio biológico do solo (Correia, 2002; Parron et al., 2015), de modo a possibilitar mudanças nas características químicas, físicas e biológicas no ecossistema, afetando as interações solo-planta (Parron et al., 2015). Por meio do estudo da fauna edáfica pode-se além de avaliar a qualidade do solo, ter uma maior compreensão do sistema de produção que este solo comporta, visto que a mesma 
está intimamente relacionada aos processos de ciclagem de nutrientes e decomposição da matéria orgânica na interface soloplanta.

No Brasil já existem diversas Unidades Industriais que processam toda a biomassa da cana para fabricar álcool de segunda geração e/ou gerar energia elétrica, tornando-se assim, uma nova fonte de renda das Usinas e Destilarias. Ao processar toda a biomassa gerada pela cana-de-açúcar, os possíveis efeitos favoráveis sobre as propriedades químicas, físicas e biológicas do solo serão retardados. Desse modo, avaliar os efeitos em relação à fertilidade do solo e a fauna edáfica são imprescindíveis para verificar se é plausível que se faça uma redução na quantidade de palha deixada no campo, a fim de esclarecer a sua atuação nos fatores edáficos. Esta revisão tem como objetivo, enfatizar o uso da fauna do solo como bioindicadora da qualidade do solo, além de apontar os efeitos da remoção parcial ou total.

\section{Metodologia}

O presente estudo é caracterizado como uma pesquisa bibliográfica, qualitativa e exploratória. A pesquisa exploratória é aquela que visa proporcionar uma maior familiaridade com o problema de um tema, ainda pouco conhecido. A bibliográfica, se baseia em produções acadêmicas ou técnicas, como livros, artigos, monografias, dissertações e/ou teses, e a qualitativa, permite que os pesquisadores usem a criatividade e imaginação, propondo uma nova ótica para o trabalho (Gil, 2008). Para tal estudo, foi realizado uma vasta procura por estudos que relatam a representatividade do setor sulcroalcooleiro, passando pela significância da implementação de novas tecnologias como a mecanização do sistema de colheita, até os impactos dessa atividade na qualidade do solo, sobretudo se tratando das interações que ocorrem entre a fauna do solo, fertilidade do solo e o manejo da palhada sob o solo. As palavras-chave cana-de-açúcar, manejo de palhada, fauna do solo, biodiversidade, sustentabilidade e suas associações foram usadas como termos de pesquisa no banco de dados ISI Web of Science, Google Scholar e Scopus, sendo selecionados apenas 32 estudos, que continham informações suficientes para serem incluídos em nosso estudo. Incluímos artigos que apresentavam informaçães sobre a geração de energias sustentáveis, expansão e evolução da cana-de açúcar no Brasil, séries históricas da safra brasileira de cana-de-açúcar, indicadores de qualidade ambiental, manejo

e conservação do solo e indicadores edafoclimáticos. É importante destacar os estudos foram compilados e analisados, buscando a compreensão de como as estratégias e práticas adotadas no cultivo de cana-de-açúcar influenciam a atividade biológica do solo e, por vias, a ciclagem de nutrientes, um processo fundamental para garantir a sustentabilidade do sistema de produção.

\section{Resultados e Discussão}

\section{Panorama da cultura no Brasil}

O setor sucroalcooleiro brasileiro desempenha um papel importante na economia do país, destacando-se a produção de açúcar e bioenergia, especialmente o etanol. A cana-de-açúcar é considerada uma das alternativas como biocombustíveis e atualmente o aumento da demanda mundial por combustíveis oriundos de fontes renováveis, como o etanol, vem aumentando (Oliveira et al., 2016). As condições edafoclimáticas favoráveis à cana-de-açúcar, aliadas às áreas cultiváveis, fizeram do Brasil o maior produtor mundial dessa commodity e o segundo em produção de etanol (CONAB, 2020). Segundo a FIESP (2013), o aumento de investimentos em novas usinas sucroalcooleiras deve-se ao acréscimo do uso de etanol em veículos Flex Fuel e à crescente demanda de açúcar do mercado internacional, que ocorreu a partir de 2003.

Estima-se que até a safra de 2023/24 a frota flex representará $28 \%$ da frota total brasileira de veículos, o que aumentará o consumo do combustível etanol (FIESP, 2013). Esse cenário vem se consolidando com as grandes produções dos últimos anos: a safra de 2019/20 encerrou-se com 642 milhões de toneladas de cana-de-açúcar, sendo 3,6\% superior a safra anterior (2018/2019). A região sudeste permanece como maior produtora nacional, tendo o Estado de São Paulo como 
principal produtor, respondendo por cerca de $53,7 \%$ da produção brasileira de cana-de-açúcar, $46,2 \%$ de etanol e $62,6 \%$ de açúcar (CONAB, 2020). Para a safra de 2020/21 estima-se que a produção de cana-de-açúcar no Brasil seja de mais de 640 milhões de toneladas, o que representa uma pequena retração de 0,1\% referente a safra anterior (2019/20), indicando melhoras neste setor agrícola. Além disso, estima-se que serão produzidas 40 milhões de toneladas de açúcar e de 28 bilhões de litros de etanol (CONAB, 2020).

A produção de etanol na safra de 2019/20 foi a maior da história do setor sucroenergético nacional; para a safra de 2020/21 o total será de cerca de 27,8 bilhões de litros, representando uma queda de 18,3\%, fato que ocorreu como consequência da pandemia do Covid-19, que afetou o consumo interno de álcool devido ao isolamento social e diminuição da circulação de pessoas com veículos automotores (CONAB, 2020). Devido à alta eficiência tanto no processo de produção quanto no balanço energético, o etanol é uma das principais alternativas de biocombustíveis limpos de viabilidade econômica e de qualidade; além disso, a quantidade de energia renovável obtida com etanol de cana-de-açúcar no Brasil pode chegar a oito vezes a energia utilizada de combustível fóssil (Camargo \& Oliveira, 2011).

Com a sustentabilidade entrando na pauta das discussões em todo o mundo, o estudo de fontes renováveis de energia e diversificação de matrizes energéticas torna-se cada vez mais importante para a evolução do desenvolvimento sustentável. Sendo assim, a bioenergia advinda da biomassa, bagaço e palha da cana-de-açúcar se caracteriza como uma importante ferramenta de geração de energia renovável: juntos, etanol e biomassa respondem por cerca de $15 \%$ da matriz energética brasileira (Lopes \& Taques, 2016). De acordo com dados da UNICA, (2013), a produção de bioeletricidade por meio de insumos das usinas de açúcar já era responsável, no ano de 2012, pela eletricidade para consumo de cinco milhões de residências, cerca de $10 \%$ da população brasileira, e a 21 estimativa é que, até o final do ano de 2020, o consumo poderá chegar a $20 \%$ da demanda nacional.

No Brasil já existem Unidades Industriais que processam toda a biomassa da cana para fabricar álcool de segunda geração e/ou gerar energia elétrica, tornando-se assim, uma nova fonte de renda das Usinas e Destilarias. Ao processar toda a biomassa gerada pela cana-de-açúcar, os possíveis efeitos favoráveis sobre as propriedades químicas, físicas e biológicas do solo serão retardados. Desse modo, aferir sobre os efeitos em relação a fertilidade do solo e sobre a fauna epígea são imprescindíveis para verificar se é plausível que se faça uma redução na quantidade de palha deixada no campo, afim de esclarecer a sua atuação nos fatores edáficos. Isto irá permitir atos de gestão que levem a preservar os seus efeitos benéficos e, ao mesmo tempo, contemplar a fabricação de álcool de segunda geração e a co-geração de energia. Considerando o exposto, e as mudanças que ocorreram no setor sucroenergético nas últimas décadas, principalmente no fato das usinas de açúcar se transformarem em indústrias não só de alimentos como também de produção de energia, como a produção do biocombustível etanol e da bioeletricidade, a necessidade de estudos sobre a produção de cana-de-açúcar e suas implicações à qualidade do solo, está cada vez mais evidente e presente na preocupação dos produtores agrícolas, e deve ser explorado de forma essencial para garantir um sistema sustentável de produção da cultura.

\section{Sistema de Colheita Mecanizado da Cana-de-Açúcar}

O sistema de colheita da cana-de-açúcar (Saccharum officinarum spp) sem queima de palhada (cana crua ou mecanizada) durante a última década vem sendo difundido. Em 2007, o Governo de São Paulo e as Usinas de cana-de-açúcar, firmaram um Protocolo Ambiental, que permitiu uma rápida penetração da colheita mecanizada no estado, eliminando a queima da palha e reduzindo a necessidade de mão de obra não qualificada (Torquato \& Ramos, 2012).

A partir desse novo cenário, o manejo da cana-de-açúcar tem sido ajustado, buscando de forma adequada atender as necessidades da cultura pleiteando uma produção satisfatória (Aquino et al., 2018; Schultz et al., 2010). Entre as razões para a mudança, da colheita manual para a colheita mecânica, estão os benefícios que a colheita mecanizada promove como a redução 
de custos para esta operação e maior produtividade do trabalho (Santoro et al., 2017). Oliveira (2013), observou em seu estudo que, o custo com a colheita mecanizada chega ser 30 a $40 \%$ inferior à colheita manual. Mas, é principalmente por questões ambientais que este processo tem se acelerado a cada ano, devido aos diversos problemas causados pela queima dos canaviais ao meio ambiente.

A palhada que é depositada na superfície do solo, desempenha um papel fundamental na sustentação da biodiversidade do solo, servindo como fonte primária de carbono (energia), protegendo o solo da radiação solar direta, regulando a temperatura e a umidade do solo e fornecendo abrigo e habitat para a fauna do solo (Benazzi et al., 2013; Menandro et al., 2019).

A quantidade de palhada acumulada no sistema sem queima da palhada varia de 10 a $30 \mathrm{t} \mathrm{ha}^{-1}$ ano-1 de material seco, que contribui para o aumento da quantidade de matéria orgânica e nutrientes no solo (Oliveira, 1999; Trivelin, 1996; Aquino et al., 2018). Sobre os atributos físicos do solo sob cultivo de cana crua, o sistema cana crua com incorporação de palhada tem contribuído para o aumento do teor de matéria orgânica, maior da estabilidade dos agregados e menores condições da densidade no solo. No entanto, Esteban et al. (2019), afirmam que o tráfego de maquinário agrícola no sistema de colheita mecanizada, aumenta a densidade do solo, tornando-o compactado. Apesar da baixa taxa de mineralização da palhada, pode-se destacar como benefícios a longo prazo a manutenção da fauna do solo, o aumento do teor de matéria orgânica no solo, a menor perda de umidade, diminui a susceptibilidade à erosão. Altas taxas de remoção de palha induzem degradação da qualidade física e biológica do solo (Castioni et al., 2018). Já o acúmulo de palhada causa no solo alterações de ordem física e biológica, melhorando a fertilidade do solo sob essas condições (Ceddia, 1996; Segnini et al., 2013).

Em relação a influência do manejo da palhada sobre a produção da cana-de-açúcar, (Aquino et al., 2018), observaram que o manejo da cana-de-açúcar por meio da queima da palha antes da colheita reduz a produtividade da cana em $49 \%$ e quando a palhada é manejada sobre a superfície do solo, a sua retirada total ou $75 \%$ da palhada do campo reduz a produção da cana em cerca de $47 \%$. O manejo de $50 \%\left(15 \mathrm{t} \mathrm{ha}^{-1}\right)$ da palhada sobre o solo é suficiente para fornecer $47 \%$ de aumento na produção em condições de déficit hídrico, e $50 \%$ do excesso de palha pode ser removido do campo para processos industriais sem afetar adversamente a safra e a produtividade. E as propriedades biológicas do solo? Será que $50 \%$ da palhada sobre o solo é suficiente para preservar os organismos no solo? (Franco et al., 2016), chama atenção pela perda significativa da biodiversidade de organismos do solo, devido a expansão das lavouras de cana nos trópicos.

Mudanças no uso da terra, como a substituição de áreas de pastagens por lavouras de cana-de-açúcar, podem causar uma redução de cerca de $90 \%$ na abundância da macrofauna do solo, além da perda de aproximadamente de $40 \%$ da diversidade dos grupos da macrofauna (Franco et al., 2016).

As propriedades físicas e químicas do solo respondem de forma lenta às mudanças no uso e no manejo do solo, ao contrário das propriedades biológicas e bioquímicas que são mais sensíveis as mudanças em curto prazo, sendo dessa forma, importantes indicadores da qualidade do solo, principalmente em avaliações de manejo do solo (Roy et al., 2018).

\section{Fauna edáfica}

O Brasil é reconhecido mundialmente pela sua vasta biodiversidade, sendo a fauna edáfica um notável componente dessa diversidade. Embora, sua maior parte apresentar-se pouco visível ou até mesmo invisível à olho nu em decorrência do seu tamanho (Figura 1) e por estar dentro do solo ou da serapilheira, esta fauna invertebrada do solo origina importantes funções ambientais (Melo et al., 2009; Souza et al., 2016). Estes organismos que compõem a fauna edáfica, possuem um importante papel nos processos de ciclagem de nutrientes, incorporação e decomposição da matéria orgânica, melhoria da agregação, sequestro de carbono, porosidade, aeração e infiltração de água no solo (Frouz, 2018; Paz-ferreiro; Fu, 2016). Os organismos que compõe a fauna edáfica são classificados conforme seu tamanho e diâmetro corporal (Figura 1). De acordo 
com Baretta et al. (2011), a microfauna do solo possui diâmetro corporal variando de $4 \mu \mathrm{m}$ a $100 \mu \mathrm{m}$, e é constituída por organismos como protozoários, bactérias e fungos. A mesofauna inclui organismos entre $100 \mu \mathrm{m}$ e $2 \mathrm{~mm}$ que se movimentam em fissuras, poros e na interface do solo, como os ácaros, collembolas, proturas e pequenos insetos. E a macrofauna é composta por invertebrados com mais $2 \mathrm{~mm}$ de diâmetro corporal, a maior parte desses organismos pode ser encontrada na camada superficial do solo (0-10 cm de profundidade), sendo esta, a camada mais afetada pelas práticas de manejo (Baretta et al., 2011; Grandy et al., 2016; Trentini et al., 2018).

Figura 1. Classificação dos diferentes grupos de organismos com base no seu tamanho.

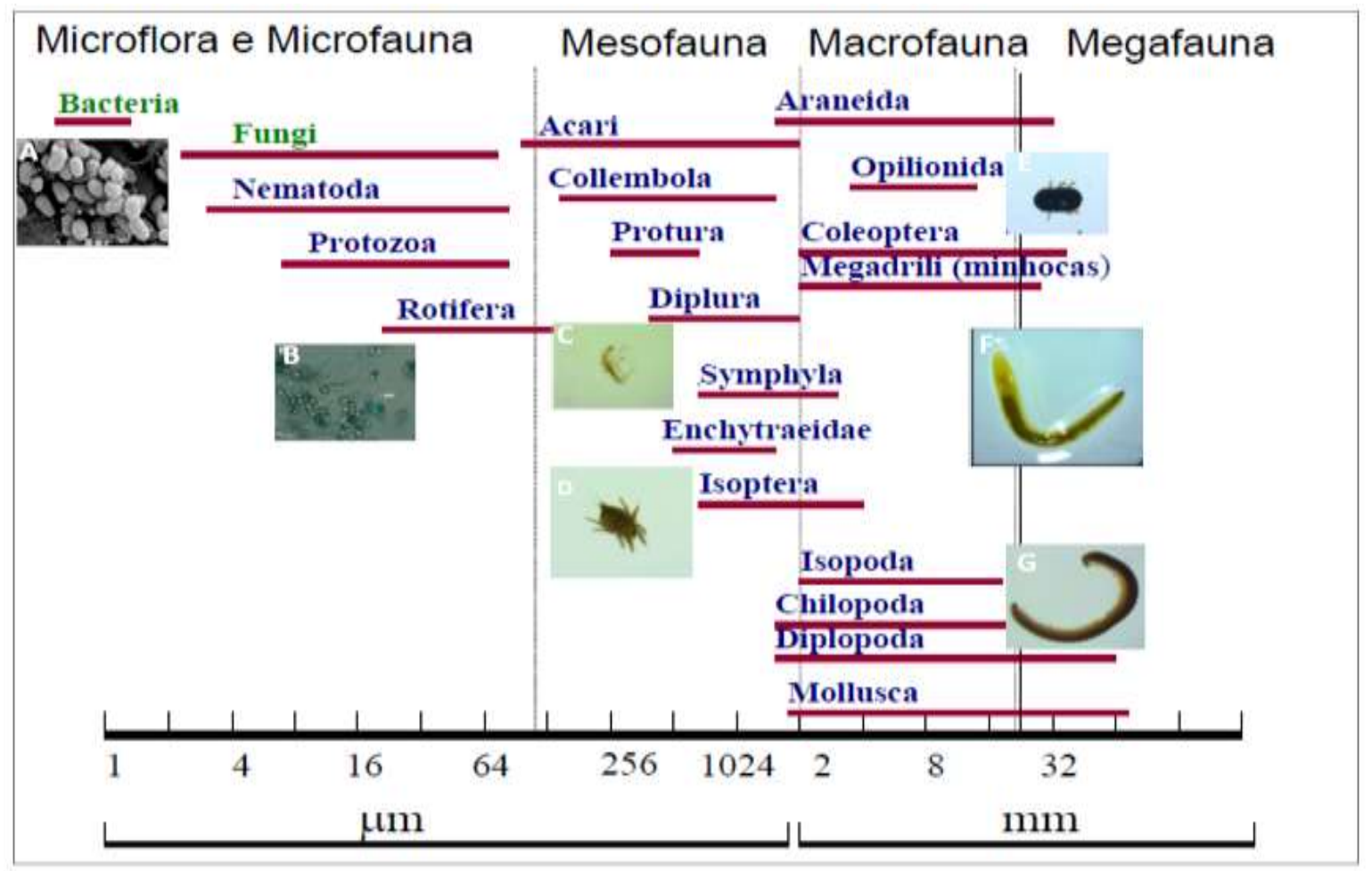

Fonte: Classificação de tamanho da biota do solo (SWIFT et al., 1979), adaptado de Corrêia \& Oliveira (2002). A Rizobactérias, fonte: https://www.agencia.cnptia.embrapa.br/; B - Fungos Micorrízicos, fonte: Gonçalves et. al. (2014); C - Collembola, D - Acari, E - Coleoptera, F - Megadrili e G - Diplopoda, fonte: (Pereira et al., 2018).

A macrofauna edáfica é composta principalmente por organismos pertencentes as classes: Gastropoda (caracóis e lesmas), Oligochaeta (minhocas), Malacostraca (tatuzinhos), Insecta (cupins, grilos, baratas, besouros, moscas, formigas e etc.), Arachnida (aranhas, opiliôes e escor) e Myriapoda (classes piôes), Chilopoda(centopeias) e Diplopoda (gongolos) ( Souza et al., 2016; Pereira et al., 2017).

As minhocas, cupins e formigas são considerados “engenheiros do solo” (Lavelle, 1997), por realizarem a bioturbação. O fenômeno de bioturbação pode ser definido como modificações da estrutura física do solo, em decorrência da formação de estruturas biogênicas (túneis, poros, agregados, coprólitos, e ninhos) pela atividade e movimentação de partículas ao longo do perfil do solo (Baretta et al., 2011). A influência mútua entre os processos de decomposição da matéria orgânica, ciclagem de nutrientes e bioturbação são responsáveis pelo balanço entre o estoque de carbono no solo e a emissão de gases de efeito estufa (Swift, 2010; Mendes, 2018). Desse modo, os organismos do solo têm um importante papel na regulação da composição da atmosfera e nas mudanças climáticas.

Algumas propriedades do solo, como aeração, drenagem, resistência à erosão, infiltração, capacidade de retenção de água e estabilidade de agregados são definidas de acordo com a intensidade em que a bioturbação ocorre no solo (Almeida et 
al., 2017). Estes organismos são capazes de indicar características físico-químicas (Velásquez et al., 2012) e estruturais do ambiente em que se encontram (Souza et al., 2016).

As informações adquiridas com a investigação da macrofauna edáfica podem cooperar para um eficiente monitoramento estratégico para recuperação do solo ou mitigação de danos causados ao solo pelo manejo empregado (Rovedder et al., 2009). De acordo com Melo et al. (2009) a abundância e a diversidade da macrofauna edáfica podem ser influenciadas pela cobertura do solo, topografia, clima, fatores edáficos (minerais, matéria orgânica, umidade, estrutura, textura e tipo de solo) e fatores históricos (geológicos e humanos), o qual corrobora com o estudo de (Lavelle, 1997).

De acordo com Franco et al. (2016), em estudo para compreender os impactos da alteração da biodiversidade e composição subterrânea no funcionamento do solo e no desempenho agrícola, avaliando a macrofauna do solo, observaram queda na abundância da macrofauna (89 \%) e na diversidade da macrofauna (39\%), quando a pastagem era substituída pela cana-de-açúcar. Franco et al. (2016) verificaram em seus respectivos estudos que a abundância e diversidade da comunidade da macrofauna está diretamente relacionada aos atributos químicos do solo. Tais mudanças alteram a diversidade e composição da fauna edáfica, com impacto crítico no funcionamento do solo (John et al., 2021). Nesse contexto, fica evidente a importância dos organismos da fauna do solo, bem como sua relação com serviços ecossistêmicos (Figura 2).

A redução ou extinção de alguns grupos da fauna invertebrados do solo e a subsequente perda de suas atividades benéficas contribuem para as altas taxas de deterioração da terra, declínio da fertilidade, redução de nutrientes e aumento de pragas de artrópodes (Bedano et al., 2016). Entre esses invertebrados do solo, a macrofauna do solo desempenha um papel importante na fragmentação e da serapilheira e consequentemente na manutenção da qualidade do solo, sendo assim, a manutenção da palhada sobre o solo pode promover a diversidade e abundância da macrofauna e melhorar a qualidade do solo (Pant et al., 2017).

Figura 2. Relação entre as atividades da fauna do solo, as características e processos edáficos importantes para a geração de serviços ambientais.

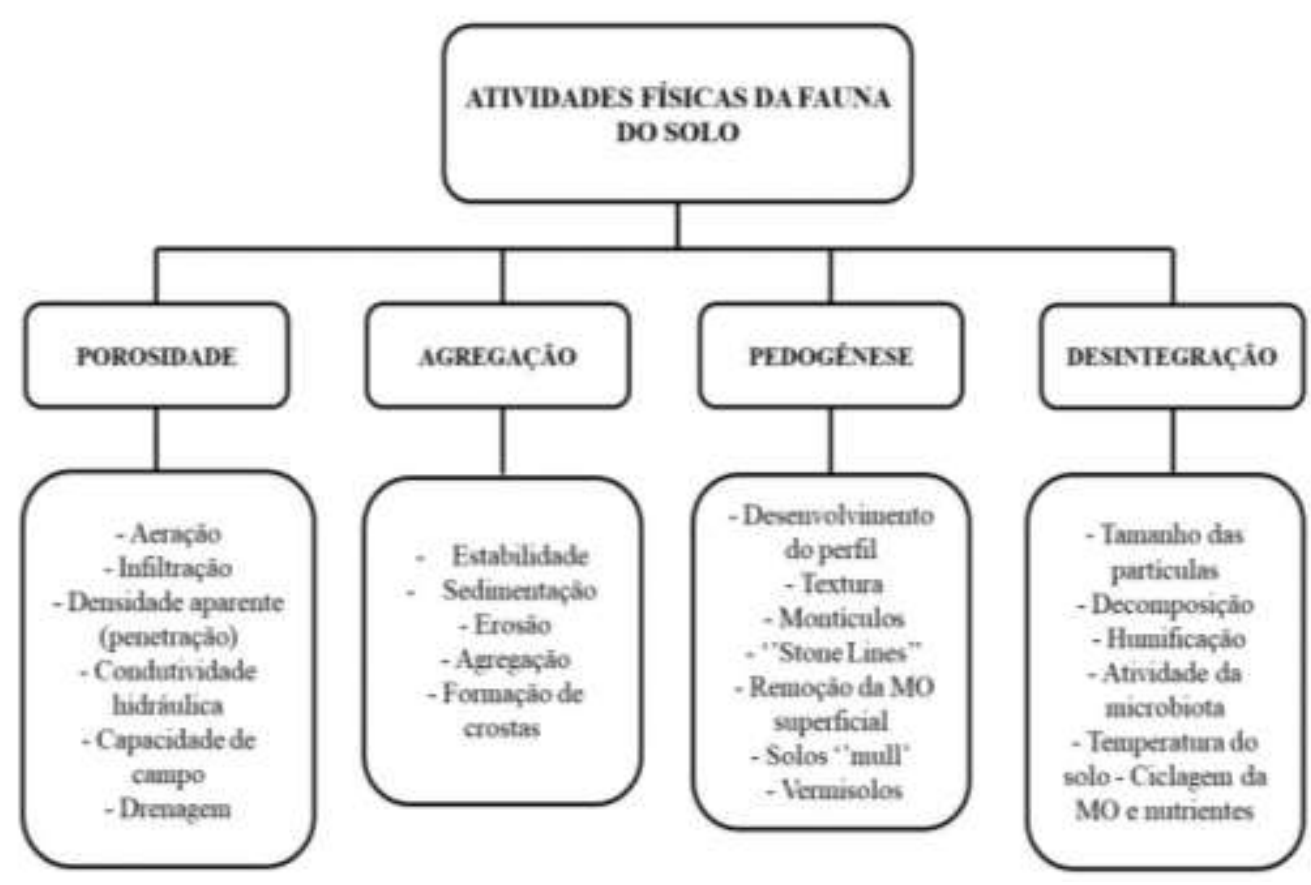

Fonte: Adaptação de (Parron et al., 2015). 


\section{Fauna edáfica como bioindicadora de qualidade do solo}

A qualidade do solo não se define somente pelo seu potencial produtivo, refere-se também à qualidade ambiental, a sanidade do homem, da fauna e da flora. A capacidade do solo em promover atividade biológica, favorecer o fluxo de água, conservar a qualidade do ambiente e operar sob efeito tampão, assimilando detritos orgânicos e outros, como Larson; Pierce (1994) definem o termo qualidade do solo.

$\mathrm{Na}$ literatura, a menção científica mais antiga e que definiu a qualidade do solo foi a proposta por Mausel (1971), no qual referiu-se à qualidade do solo como "a capacidade do mesmo em produzir trigo, soja e milho sob condições de manejo do solo", considerando apenas as culturas que dominavam a economia na época. No entanto, Doran e Parkin (1994) observaram que esta definição não comtemplava a capacidade do solo de funcionar de forma sustentável e, então, incluíram a contribuição ambiental e promoção da saúde das plantas nela. A qualidade do solo é a base para o desenvolvimento sustentável do uso antrópico da terra e a preocupação em sua manutenção sob cultivo de cana-de-açúcar tem aumentado nos últimos anos.

A degradação na qualidade do solo ao longo dos anos é resultado do aumento do uso de recursos naturais associada à expansão agrícola feita pelo homem. Os solos variam naturalmente em qualidade em função de suas propriedades e, dependendo do tipo de manejo utilizado, podem ocorrer mudanças em seus atributos físicos, químicos e biológicos (Chaves et al., 2017). Os indicadores de qualidade do solo são os atributos físicos, químicos e biológicos que refletem a capacidade de funcionamento do solo e seu estado atual. Segundo Simões et al. (2018), os indicadores físicos mais utilizados são a resistência do solo à penetração, porosidade total do solo, densidade do solo e a estabilidade de seus agregados. Para Bunemann et al. (2018), a matéria orgânica e o pH são os mais propostos, seguidos pelo fósforo disponível para absorção das plantas, densidade do solo e alguns indicadores de armazenamento de água. Cherubin et al. (2015) utilizaram os indicadores químicos pH, fósforo disponível e potássio; o físico foi representado pela densidade do solo e o carbono orgânico e biomassa microbiana como biológicos. Desse modo, conclui-se que os atributos físicos, químicos e biológicos do solo são potenciais indicadores de sua qualidade, podendo ser verificados por meio das alterações promovidas pelos diferentes sistemas de manejo utilizados, bem como identificar quais atributos não são sensíveis a longo prazo, ou aqueles pouco sensíveis aos efeitos de preparo e manejo agrícola utilizado (Castioni et al., 2018) (Castioni et al., 2018; Cherubin et al., 2015).

Os bioindicadores são definidos como organismos que apresentam alta responsividade à efeitos negativos neles mesmos e em suas respectivas comunidades e ecossistemas (Correia, 2002; Manhaes \& Francelino, 2012).

Os organismos da fauna edáfica podem responder rapidamente sobre a qualidade do solo, uma vez que de maneira peculiar, são sensíveis às mudanças, com consequente, adequação às condições ambientais, particularidade esta que não se aplica aos indicadores químicos ou físicos (Melo et al., 2009). A fauna edáfica por estar fortemente relacionada os processos de decomposição e ciclagem de nutrientes, na interface solo-planta colabora proeminentemente com a avaliação da qualidade de um solo e a dinâmica do sistema produtivo (Parron et al., 2015).

A sensibilidade desses organismos às alterações no solo e na cobertura vegetal, decorrentes de atividades antrópicas ou distúrbios naturais, possibilita seu uso como bioindicadores da qualidade do solo ou da fertilidade (Melo et al., 2009). Os distúrbios ambientais alteram a fauna edáfica conforme alteram a disponibilidade de recursos alimentares, afetando as relações ecológicas intra e interespecíficas. Várias espécies podem desaparecer, como consequência do desmatamento e da mecanização no preparo do solo (Santoro et al., 2017). Portanto o uso e manejo do solo estão diretamente relacionados a presença e diminuição de grupos da biodiversidade do solo (Figura 3).

No estudo de Aquino et al., 2018, os autores observaram que o manejo da cana-de-açúcar por meio da queima da palha antes da colheita reduz a produtividade da cana em $49 \%$ e quando a palhada é manejada sobre a superfície do solo, a sua retirada total ou $75 \%$ da palhada do campo reduz a produção da cana em cerca de $47 \%$. 
Figura 3. Perda significativa da biodiversidade por consequências de mudanças de uso de terra, para expansão da cultura de cana-de-açúcar.

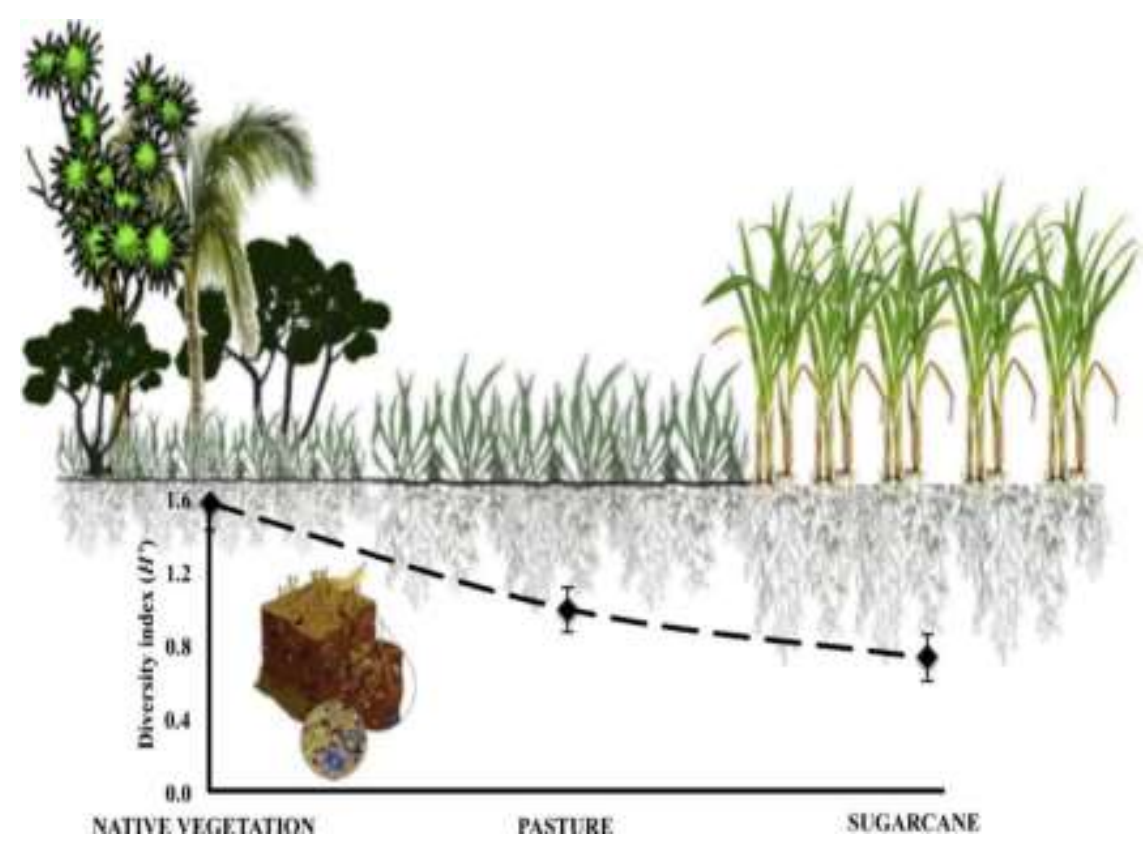

Fonte: Franco et al. (2016).

\section{Correlações entre o manejo da cana-de-açúcar e a fauna edáfica}

As práticas agrícolas e sistemas de cultivo simplificados, como o cultivo da cana-de-açúcar em monocultura, podem afetar a abundância e diversidade da macrofauna (Abreu et al.,2014; Benazzi et al., 2013; Franco et al., 2016; Menandro et al., 2019). A cultura de cana-de-açúcar tem grande importância no setor econômico, social e ambiental do estado de São Paulo e do Brasil sendo estratégica para a produção bioenergética nacional e mundial devido à crescente demanda energética (Goldemberg et al, 2014). Porém, quando tratamos de aspectos biológicos do solo, podem haver impactos negativos na biodiversidade, os quais ameaçam a sustentabilidade do cultivo da cana no Brasil.

A pesquisa voltada a biodiversidade do solo e suas funções têm crescido, visando o desenvolvimento de práticas sustentáveis de manejo do solo (Joly et al., 2018). Recentemente, têm-se realizado estudos voltados para os impactos das mudanças no uso da terra sobre a perda ou ganho de habitats da biodiversidade do solo. Franco et al. (2016) verificaram que a introdução da cana-de-açúcar sobre áreas de pastagem promoveu uma redução de abundância (89\%) e diversidade (39\%) da macrofauna do solo. Essa redução deve-se principalmente às intensas práticas de preparo do solo e a adoção de insumos agrícolas no sistema da cana-de-açúcar (Franco et al., 2016; Oliveira et al., 2019). Portilho et al., (2011), avaliando os efeitos de cultivos de cana-de-açúcar, com e sem queima de palhada na colheita, sobre parâmetros ecológicos da fauna invertebrada epigeica (mesofauna) no Município de Dourados-MS, observou que no sistema de colheita mecanizada (sem queima da palhada da cana-de-açúcar) favoreceu o desenvolvimento da fauna invertebrada, sendo indicado pelo acréscimo da diversidade, riqueza e densidade de indivíduos. Do mesmo modo, (Lima et al., 2020), concluíram em seu estudo que a colheita da cana crua e a manutenção da palhada sobre o solo, proporcionou uma maior oferta de alimento contribuindo para uma maior riqueza e diversidade de organismos.

A redução da macrofauna nos solos da cana-de-açúcar pode afetar atributos físicos e químicos do solo. Grupos como formigas, cupins e minhocas desempenham um papel fundamental na estruturação e estabilização dos solos. Participam da agregação e aumento da macroporosidade do solo, além de criar hábitats para organismos, incluindo microrganismos e planta (Culliney, 2013; Lavelle et al., 2006). Franco et al. (2020), verificaram que os engenheiros do ecossistema afetam a 
estruturação do solo, proteção física da MOS e finalmente o estoque de C do solo. Nesse sentido, a redução da diversidade da macrofauna ou abundância de grupos específicos tem efeito direto e indireto em importantes funções e serviços ecossistêmicos do solo como sequestro de $\mathrm{C}$, decomposição, ciclagem de nutriente, estruturação do solo, regulação do fluxo de água e produtividade das plantas (Bottinelli et al, 2015; Menta \& Remelli 2020).

O cultivo intensivo, caracterizado pela mecanização das principais etapas da produção, como o preparo do solo e a colheita da cultura da cana-de-açúcar, pode apresentar efeitos negativos aos atributos físicos e biológicos como maior compactação do solo, degradação estrutural e menor armazenamento de carbono orgânico do solo que estão intimamente ligados a menor abundância e diversidade da macrofauna do solo (Cherubin et al., 2016). Além disso, remoção de resíduos vegetais sobre a superfície do solo pode potencializar o efeito negativo da cana-de-açúcar na abundância da macrofauna (Menandro et al., 2019). Estudos revelaram que a remoção total da palhada do solo prejudica a macrofauna, resultando na redução da abundância, riqueza e diversidade da macrofauna do solo (Menandro et al., 2019; Portilho et al., 2011). Sistemas onde há presença de resíduos vegetais sobre o solo favorecem maior abundância de grupos de predadores, em relação a sistemas em que não há cobertura (Lima et al., 2020; Silva et al, 2008).

A deposição da palha na superfície do solo contribui para entrada de carbono no solo e, diversificação de habitat tornando local heterogêneo, de preferência para os organismos de superfície, como abrigo, nidificação, alimentação (Portilho et al., 2011). Segundo Moço et al. (2005), a maior ou menor associação de determinados grupos da fauna edáfica se deve ao tipo de preparo do solo e aos resíduos vegetais mantidos na superfície do solo, proporcionando um ambiente favorável à sobrevivência destes grupos. Aguiar e Bührnheim (2011), abordam que a representatividade de alguns táxons está relacionada a disponibilidade de alimento na área, principalmente para os predadores como Pseudoscorpionida, Araneae e Hymenoptera que podem ser atraídos pela presença de presas no local. Sendo um indicativo de estabilidade do ecossistema pela manutenção das cadeias alimentares (Benazzi et al., 2013).

\section{Considerações Finais}

A biodiversidade do solo é fundamental para processos ao nível de ecossistema, multifuncionalidade e serviços de ecossistema. As funções do ecossistema, como decomposição e mineralização de nutrientes, são desempenhadas, em parte, por um conjunto bem diverso da fauna do solo, classificados de acordo com o seu tamanho em microfauna, mesofauna e macrofauna, nos quais cada grupo compõem suas importantes funções.

Esses organismos possuem alta sensibilidade as mudanças do ambiente, por isso o monitoramento da comunidade de fauna edáfica torna-se uma ferramenta importante para a compreensão da dinâmica ecológica dos ambientes proporcionando, um manejo mais adequado de sistemas agrícolas, principalmente a área canavieira, onde atualmente se discute a quantidade de palha ideal que deve ser retirada do solo. Isto irá permitir atos de gestão que levem a preservar os seus efeitos benéficos e, ao mesmo tempo, contemplar a fabricação de álcool de segunda geração e a co-geração de energia. Outro ponto à destacar é que novos estudos a cerca do manejo do solo, e respectivas tecnologias implementadas para o otimização da produção sulcroalcooleira e suas interações com a qualidade do solo serão imprescindíveis para que seja resguardada a sustentabilidade dessa atividade, dada a necessidade atual de buscar soluções inovadoras para produção agrícola aperfeiçoada em uma gestão eficiente dos recursos naturais.

\section{Referências}

Abreu, R. R. L., Lima, S. S., Oliveira, N. C. R., Leite, L. F. C. (2014). Fauna edáfica sob diferentes níveis de palhada em cultivo de cana-de-açúcar. Pesquisa Agropecuária, 44(4), 409-416.

Aguiar, N. O., Bührnheim, P. F. (2011). Pseudoscorpionida (Arachnida) em galerias de colônias de Passalidae (Coleoptera, Insecta) em troncos caídos em floresta de terra firme da Amazônia, Brasil. Acta Amazônica, 41(2), 311-320. 
Aguilera Esteban, D. A., de Souza, Z. M., Tormena, C. A., Lovera, L. H., de Souza Lima, E., de Oliveira, I. N., \& de Paula Ribeiro, N. (2019). Soil compaction, root system and productivity of sugarcane under different row spacing and controlled traffic at harvest. Soil and Tillage Research, 187, 60-71. https://doi.org/10.1016/j.still.2018.11.015

Almeida, H. S., da Silva, R. F., Grolli, A. L., \& Schied, D. L. (2017). Ocorrência e diversidade da fauna edáfica sob diferentes sistemas de uso do solo. Revista Brasileira De Tecnologia Agropecuária, 1(1), 15-23.

Aquino, A. M. de, \& Correia, M. E. F. (2005). Invertebrados edáficos e o seu papel nos processos do solo. Embrapa Agrobiologia. Documentos, 201, 1-52.

Aquino, G. S. de, de Conti Medina, C., Shahab, M., Santiago, A. D., Cunha, A. C. B., Kussaba, D. A. O., Carvalho, J. B., \& Moreira, A. (2018). Does straw mulch partial-removal from soil interfere in yield and industrial quality sugarcane? A long term study. Industrial Crops and Products, 111, 573-578. https://doi.org/10.1016/j.indcrop.2017.11.026

Baretta, D., Santos, J. C. P., Segat, J. C., \& Geremia, E. V. (2011). Fauna edáfica e qualidade do solo. Tópicos Em Ciência Do Solo, October 2014, 119-170.

Bedano, J. C., Domínguez, A., Arolfo, R., \& Wall, L. G. (2016). Effect of Good Agricultural Practices under no-till on litter and soil invertebrates in areas with different soil types. Soil and Tillage Research, 158, 100-109. https://doi.org/10.1016/j.still.2015.12.005

Benazzi, E. S., Bianchi, M. O., Correia, M. E. F., Lima, E., Zonta, E. (2013). Impactos dos métodos de colheita da cana-de-açúcar sobre a macrofauna do solo em área de produção no Espírito Santo - Brasil. Semina: Ciências Agrárias, 34(6), 3425-3442.

Bottinelli, N., Jouquet, P., Capowiez, Y., Podwojewski, P., Grimaldi, M., \& Peng, X. (2015). Why is the influence of soil macrofauna on soil structure only considered by soil ecologists? Soil and Tillage Research, 146, 118-124. https://doi.org/https://doi.org/10.1016/j.still.2014.01.007

Bueno, O., \& Morini, M. (2017). Cana-de-açúcar e seus impactos: uma visão acadêmica. April 2018.

Bunemann, E. K., Bongiorno, G., Bai, Z., Creamer, R. E., Deyn, G. D., Goede, R., Fleskens, L., Geissen, V., Kuyper, T. W., Mäder, P., Pulleman, M., Sukkel, W., Groenigen, J. W. V., Brussaard, L. (2018). Soil quality - A critical review. Soil Biology and Biochemistry, 120(1), 105-125.

Camargo, A. S., Oliveira, M. M. B. (2011). Eficiência econômica no setor sucroalcooleiro: uma análise de algumas usinas do estado de São Paulo. Organizações Rurais \& Agroindustriais, 13(3), 330-343.

Carvalho, J. L. N., Hudiburg, T. W., Franco, H. C. J., \& DeLucia, E. H. (2017). Contribution of above- and belowground bioenergy crop residues to soil carbon. GCB Bioenergy, 9(8), 1333-1343. https://doi.org/10.1111/gcbb.12411

Castioni, G. A., Cherubin, M. R., Menandro, L. M. S., Sanches, G. M., Bordonal, R. de O., Barbosa, L. C., Franco, H. C. J., \& Carvalho, J. L. N. (2018). Soil physical quality response to sugarcane straw removal in Brazil: A multi-approach assessment. Soil and Tillage Research, 184, 301-309. https://doi.org/10.1016/j.still.2018.08.007

Cerri, C. E. P., Galdos, M. V., Carvalho, J. L. N., Feigl, B. J., \& Cerri, C. C. (2013). Quantifying soil carbon stocks and greenhouse gas fuxes in the sugarcane agrosystem: Point of view. Scientia Agricola, 70(5), 361-368. https://doi.org/10.1590/S0103-90162013000500011

Chaves, H. M. L., Lozada, C. M. C., Gaspar, R. O. (2017). Soil quality index of on Oxisol under different land in the Brazilian savannah. Geoderma, 10(1), $183-190$.

Cherubin, M. R., Tormena, C. A., Karlen, D. L. (2017). Soil Quality Indexing Strategies for Evaluating Sugarcane Expansion in Brazil. Revista Brasileira de Ciencia Do Solo, 41(1), 1-19.

Cherubin, M. R., Eitelwein, M. T., Fabbris, C., Weirich, S. W., Silva, R. F., Silva, V. R., Bassp, C. J. (2015). Qualidade física, química e biológica de um latossolo com diferentes manejos e fertilizantes. Revista Brasileira de Ciência Do Solo, 39(2), 615-625.

Cherubin, M. R., Karlen, D. L., Franco, A. L. C., Cerri, C. E. P., Tormena, C. A., \& Cerri, C. C. (2016). A Soil Management Assessment Framework (SMAF) Evaluation of Brazilian Sugarcane Expansion on Soil Quality. Soil Science Society of America Journal, 80(1), 215-226. https://doi.org/10.2136/sssaj2015.09.0328

CONAB. (2020). Companhia Nacional de Abastecimento. Safra Brasileira de Cana-de-Açúcar 2020.

Correia, M. E. F. (2002). Relações entre a Diversidade da Fauna de Solo e o Processo de Decomposição e seus Reflexos sobre a Estabilidade dos Ecossistemas. Seropédica: Embrapa Agrobiologia, Documentos 156, 33.

Culliney, T. (2013). Role of Arthropods in Maintaining Soil Fertility. Agriculture, 3(4), 629-659. https://doi.org/https://doi.org/10.3390/agriculture3040629

De Souza, S. T., Cassol, P. C., Baretta, D., Bartz, M. L. C., Klauberg Filho, O., Mafra, Á. L., \& Da Rosa, M. G. (2016). Abundance and diversity of soil macrofauna in native forest, eucalyptus plantations, perennial pasture, integrated crop-livestock, and no-tillage cropping. Revista Brasileira de Ciencia Do Solo, 40, 1-14. https://doi.org/10.1590/18069657rbcs20150248

FIESP. (2013). Outlook Fiesp 2023: projeções para o agronegócio brasileiro. 37-45.

Franco, A. L. C., Cherubin, M. R., Cerri, C. E. P., Six, J., Wall, D. H., \& Cerria, C. C. (2020). Linking soil engineers, structural stability, and organic matter allocation to unravel soil carbon responses to land-use change. Soil Biology and Biochemistry, 107998. https://doi.org/https://doi.org/10.1016/j.soilbio.2020.107998

Franco, A. L. C., Bartz, M. L. C., Cherubin, M. R., Baretta, D., Cerri, C. E. P., Feigl, B. J., Wall, D. H., Davies, C. A., \& Cerri, C. C. (2016). Loss of soil (macro)fauna due to the expansion of Brazilian sugarcane acreage. Science of the Total Environment, 563-564, 160-168. https://doi.org/10.1016/j.scitotenv.2016.04.116 
Frouz, J. (2018). Effects of soil macro- and mesofauna on litter decomposition and soil organic matter stabilization. Geoderma, 332, 161-172. https://doi.org/10.1016/j.geoderma.2017.08.039

GIL, A. C. (2008). Como elaborar projetos de pesquisa (4a ed.). Atlas.

Goldemberg, J., Mello, F. F. C., Cerri, C. E. P., Davies, C. A., \& Cerri, C. C. (2014). Meeting the global demand for biofuels in 2021 through sustainable land use change policy. Energy Policy, 69, 14-18. https://doi.org/https://doi.org/10.1016/j.enpol.2014.02.008

Grandy, A. S., Wieder, W. R., Wickings, K., \& Kyker-Snowman, E. (2016). Beyond microbes: Are fauna the next frontier in soil biogeochemical models? Soil Biology and Biochemistry, 102, 40-44. https://doi.org/10.1016/j.soilbio.2016.08.008

John, K., Zaitsev, A. S., \& Wolters, V. (2021). Soil fauna groups respond differentially to changes in crop rotation cycles in rice production systems. Pedobiologia, 84, 150703. https://doi.org/10.1016/j.pedobi.2020.150703

Joly, C. A., G Padgurschi Autores, M. C., F Pires, A. P., Antonio Agostinho, A., Carlos Marques, A., Amaral, A., \& Dias Loyola, R. (2018). CAPÍTULO 1 _ Apresentando o diagnóstico brasileiro de biodiversidade e serviços ecossistêmicos. In Retrieved from.

Larson, W. E., \& Pierce, F. J. (1994). The Dynamics of Soil Quality. Defining Soil Quality for a Sustainable Environment, SSSA Spec. Publ. 35, $37-51$.

Lavelle, P., Decaëns, T., Aubert, M., Barot, S., Blouin, M., Bureau, F., \& Rossi, J. P. (2006). Soil invertebrates and ecosystem services. European Journal of Soil Biology, 42. https://doi.org/https://doi.org/10.1016/j.ejsobi.2006.10.002

Lavelle, P. (1997). Faunal Activities and Soil Processes: Adaptive Strategies That Determine Ecosystem Function. Advances in Ecological Research, 27(C), 93-132. https://doi.org/10.1016/S0065-2504(08)60007-0

Lima, S. S., Pereira, M. G., Lima, E. , Zonta, E. (2020). Funcionalidade da fauna edáfica em áreas com difrentes manejos da cana-de-açúcar:. In: Robson José de Oliveira. (Org.). Funcionalidade da fauna edáfica em áreas com diferentes manejos da cana-de-açúcar. Editora Científica, 3, 48-59.

Lopes, M. C., Taques, F. H. (2016). O desafio da energia sustentável no Brasil. Revista Cadernos Da Economia, 20(36), 71-96.

Manhaes, C. M. C., \& Francelino, F. M. A. (2012). Estudo Da Inter-Relação Da Qualidade Do Solo E Da Serapilheira Com a Fauna Edáfica Utilizando Análise Multivariada. Nucleus, 9(2), 21-31. https://doi.org/10.3738/1982.2278.701

Melo, F. V. De, Brown, G. G., Luizão, F. J., \& Zanetti, R. (2009). A importância da meso e macrofauna do solo na fertilidade e como biondicadores. Boletim Informativo Da SBCS, 1(May 2014), 38-41.

Menandro, L. M. S., de Moraes, L. O., Borges, C. D., Cherubin, M. R., Castioni, G. A., \& Carvalho, J. L. N. (2019). Soil Macrofauna Responses to Sugarcane Straw Removal for Bioenergy Production. Bioenergy Research, 12(4), 944-957. https://doi.org/10.1007/s12155-019-10053-2

Menta, C., \& Remelli. (n.d.). Soil Health and Arthropods: From Complex System to Worthwhile Investigation. Insects, 11(1), 54. https://doi.org/https://doi.org/10.3390/insects11010054

Moço, M. K. S., Gama-Rodrigues, E. F., Gama-Rodrigues, A. C., Correia, M. E. (2005). Caracterização da fauna edáfica em diferentes coberturas vegetais na região norte fluminense. Revista Brasileira de Ciência Do Solo, 29(4), 555-564.

Oliveira, D. M. S., Cherubin, M. R., Franco, A. L. C., Santos, A. S., Gelain, J. G., Dias, N. M. S., ... Cerri, C. E. P. (2019). Is the expansion of sugarcane over pasturelands a sustainable strategy for Brazil's bioenergy industry? Renewable and Sustainable Energy Reviews. https://doi.org/https://doi.org/10.1016/j.rser.2018.12.012

Oliveira, F. E. R., Oliveira, J. M., Xavier, F. A. S. (2016). Changes in soil organic carbon fractions in response to cover crops in an orange orchard. Revista Brasileira de Ciência Do Solo, 40.

Oliveira, A. P. P. (2013). Atributos Edáficos e Adubação Nitrogenada em. Curso de Pós-Graduação Em Agronomia.

Pant, M., Negi, G. C. S., \& Kumar, P. (2017). Macrofauna contributes to organic matter decomposition and soil quality in Himalayan agroecosystems, India. Applied Soil Ecology, 120, 20-29. https://doi.org/10.1016/j.apsoil.2017.07.019

Parron, L. M., Garcia, J. R., Oliveira, E. B. De, Brown, G. G., \& Prado, R. B. (2015). Biodiversidade da fauna do solo e sua contribuição para os serviços ambientais. Serviços Ambientais Em Sistemas Agrícolas e Florestais Do Bioma Mata Atlântica, 122-154.

Paz-Ferreiro, J., \& Fu, S. (2016). Biological Indices for Soil Quality Evaluation: Perspectives and Limitations. Land Degradation and Development, 27(1), 14-25. https://doi.org/10.1002/ldr.2262

Pereira, J. de M., Segat, J. C., Baretta, D., Vasconcellos, R. L. de F., Baretta, C. R. D. M., \& Cardoso, E. J. B. N. (2017). Soil macrofauna as a soil quality indicator in native and replanted Araucaria angustifolia forests. Revista Brasileira de Ciencia Do Solo, $41,1-15$. https://doi.org/10.1590/18069657rbcs20160261

Pereira, M. G., Cabreira, W., Lima, S. S. De, \& Santos, R. N. (2018). Manual de coleta e identificação da fauna edafica 2018. May.

Portilho, I. I. R., Borges, C. D., Costa, A. R., Salton, J. C., Mercante, F. M. (2011). Resíduos da cultura da cana-de-açúcar e seus efeitos sobre a fauna invertebrada epigeica. Semina: Ciências Agrárias, 32(3), 959-970.

Rosa, M. G., Filho, O. K., Bartz, M. L. C., Mafra, Á. L., De Sousa, J. P. F. A., \& Baretta, D. (2015). Macrofauna edáfica e atributos físicos e químicos em sistemas de uso do solo no planalto catarinense. Revista Brasileira de Ciencia Do Solo, 39(6), 1544-1553. https://doi.org/10.1590/01000683rbcs20150033

Rovedder, A. P. M., Eltz, F. L. F., Drescher, M. S., Schenato, R. B., Antoniolli, Z. I. (2009). Organismos edáficos como bioindicadores da recuperação de solos degradados por arenização no Bioma Pampa. Ciência Rural, 39(4), 1061-1068. 
Research, Society and Development, v. 10, n. 10, e239101018741, 2021

(CC BY 4.0) | ISSN 2525-3409 | DOI: http://dx.doi.org/10.33448/rsd-v10i10.18741

Roy, S., Roy, M. M., Jaiswal, A. K., \& Baitha, A. (2018). Soil Arthropods in Maintaining Soil Health: Thrust Areas for Sugarcane Production Systems. In Sugar Tech (Vol. 20, Issue 4, pp. 376-391). Springer India. https://doi.org/10.1007/s12355-018-0591-5

Santoro, E., Soler, E. M., \& Cherri, A. C. (2017). Route optimization in mechanized sugarcane harvesting. In Computers and Electronics in Agriculture (Vol. 141, pp. 140-146). Elsevier B.V. https://doi.org/10.1016/j.compag.2017.07.013

Schultz, N., Lima, E., Pereira, M. G., \& Zonta, E. (2010). Efeito residual da adubação na cana-planta e da adubação nitrogenada e potássica na cana-soca colhidas com e sem a queima da palhada. Revista Brasileira de Ciência Do Solo, 34(3), 811-820. https://doi.org/10.1590/s0100-06832010000300023

Segnini, A., Carvalho, J. L. N., Bolonhezi, D., Milori, D. M. B. P., da Silva, W. T. L., Simões, M. L., Cantarella, H., de Maria, I. C., \& Martin-Neto, L. (2013). Carbon stock and humification index of organic matter affected by sugarcane straw and soil management. Scientia Agricola, 70(5), 321-326. https://doi.org/10.1590/S0103-90162013000500006

Silva, R., Aquino, A., Mercante, F., \& Guimarães, M. (2008). No Title. Acta Scientiarum - Agronomy, 30(5), 725-731. https://doi.org/https://doi.org/10.1590/S1807-86212008000500018

Simões, V. J. L. P., Leite, M. L. V., Souza, E. S., Lucena, L. R. R, Izidro, J. L. P. S. (2018). Indicadores de sustentabilidade com base na qualidade do solo e acúmulo de fitomassa em pastagens degradadas. Journal Agrarian Academy, 5(9), 253-274.

Torquato, S. A., \& Ramos, R. C. (2012). Protocolo Agroambiental do setor sucroalcooleiro paulista: ações visando à preservação ambiental. Analise $e$ Indicadores Do Agronegócio, 7(6), 1.

Trentini, C. P., Villagra, M., Gómez Pámies, D., Bernava Laborde, V., Bedano, J. C., \& Campanello, P. I. (2018). Effect of nitrogen addition and litter removal on understory vegetation, soil mesofauna, and litter decomposition in loblolly pine plantations in subtropical Argentina. Forest Ecology and Management, 429 , 133-142. https://doi.org/10.1016/j.foreco.2018.07.012

UNICA. (2013). Etanol e bioletricidade: Vitais para diversificação da matriz energética nacional. UNICA - União Da Indústria De Cana-De-Açúcar. http://www.unica.com.br/noticia/3809058592036840361/etanol-e-bioeletricidade-por-cento3A-vitais-para-diversificacao-da-matriz-energetica-nacional-/

Velásquez, E., Fonte, S. J., Barot, S., Grimaldi, M., Desjardins, T., \& Lavelle, P. (2012). Soil macrofauna-mediated impacts of plant species composition on soil functioning in Amazonian pastures. Applied Soil Ecology, 56, 43-50. https://doi.org/10.1016/j.apsoil.2012.01.008 